\title{
Article $\beta$-Ketophosphonates with Pentalenofuran Scaffolds Linked to
the Ketone Group for the Synthesis of Prostaglandin Analogs
}

\author{
Constantin I. Tănase ${ }^{1, *} \mathbb{C}$, Constantin Drăghici ${ }^{2}$, Miron Teodor Căproiu ${ }^{2}$, Anamaria Hanganu ${ }^{2} \mathbb{D}$, \\ Gheorghe Borodi ${ }^{3}$, Maria Maganu ${ }^{2}$, Emese Gal $^{4}$ and Lucia Pintilie ${ }^{1}$ (i)
}

1 National Institute for Chemical-Pharmaceutical Research and Development-ICCF, 112 Vitan Av., 031299 Bucharest, Romania; lucia.pintilie@gmail.com

2 Organic Chemistry Center "C.D.Nenittescu”, 202B, Splaiul Independentei, 060023 Bucharest, Romania; cst_drag@yahoo.com (C.D.); dorucaproiu@gmail.com (M.T.C.); anamaria_hanganu@yahoo.com (A.H.); mmaganu@yahoo.com (M.M.)

3 National Institute for R\&D of Isotopic and Molecular Technologies, 67-103 Donat, 400293 Cluj-Napoca, Romania; borodi@itim-cj.ro

4 Faculty of Chemistry and Chemical Engineering, Babes-Bolyai University, Aranylános 11, 400012 Cluj-Napoca, Romania; gal.emese.81@gmail.com

* Correspondence: cvtanase@gmail.com

+ This is our 3 rd paper in the field of $\beta$-ketophosphonates. See references.

check for updates

Citation: Tănase, C.I.; Drăghici, C.; Căproiu, M.T.; Hanganu, A.; Borodi, G.; Maganu, M.; Gal, E.; Pintilie, L.

\section{$\beta$-Ketophosphonates with}

Pentalenofuran Scaffolds Linked to the Ketone Group for the Synthesis of Prostaglandin Analogs . Int. J. Mol. Sci. 2021, 22, 6787. https://doi.org/ $10.3390 /$ ijms 22136787

Academic Editor: Holger Wille

Received: 17 May 2021

Accepted: 23 June 2021

Published: 24 June 2021

Publisher's Note: MDPI stays neutral with regard to jurisdictional claims in published maps and institutional affiliations.

Copyright: (c) 2021 by the authors. Licensee MDPI, Basel, Switzerland. This article is an open access article distributed under the terms and conditions of the Creative Commons Attribution (CC BY) license (https:// creativecommons.org/licenses/by/ $4.0 /)$.

\begin{abstract}
Ketophosphonates with pentalenofurane fragments linked to the keto group were synthesized. The bulky pentalenofurane skeleton is expected to introduce more hindrance in the prostaglandin analogues of type III, greater than that obtained with the bicyclo[3.3.0]oct(a)ene fragments of prostaglandin analogues I and II, to slow down (retard) the inactivation of the prostaglandin analogues by oxidation of $15 \alpha-\mathrm{OH}$ to the 15 -keto group via the $15-\mathrm{PGDH}$ pathway. Their synthesis was performed by a sequence of three high yield reactions, starting from the pentalenofurane alcohols $\mathbf{2}$, oxidation of alcohols to acids $\mathbf{3}$, esterification of acids $\mathbf{3}$ to methyl esters $\mathbf{4}$ and reaction of the esters 4 with lithium salt of dimethyl methanephosphonate at low temperature. The secondary compounds $\mathbf{6 b}$ and $\mathbf{6 c}$ were formed in small amounts in the oxidation reactions of $\mathbf{2 b}$ and $\mathbf{2 c}$, and the NMR spectroscopy showed that their structure is that of an ester of the acid with the starting alcohol. Their molecular structures were confirmed by single crystal X-ray determination method for $6 \mathrm{c}$ and XRPD powder method for $\mathbf{6 b}$.
\end{abstract}

Keywords: $\beta$-ketophosphonates; halogeno-pentalenofurane scafold; 15 -substituted prostaglandin analogs; dimethyl methanephosphonate; X-ray crystallography; 15-bulky prostaglandin substituents

\section{Introduction}

In the prostaglandin (PG) and prostaglandin analogs (PGs), the inactivation of PGs is mainly realized by enzyme oxidation of the $15 \alpha-\mathrm{OH}$ to the 15-keto group via the 15-PGDH pathway. To slow down (retard) the $15 \alpha-\mathrm{OH}$ oxidation, some structural modifications have been done: the introduction of a 15-OH,15-methyl group [1], like in arbaprostil [2], prostalene [3], tioprostanide [4], etc., a 16-OH,16-methyl group at $\mathrm{C}_{16}$ carbon atom, like in mexiprostil [5], two methyl groups at $C_{16}$, like in nocloprost [6], gemeprost [7,8], cyclopentyl and cyclohexyl scafolds for a few prostacyclin and carbacyclin type compounds, like: ataprost [9,10], CH-5084 [11], SC-43350 [12] for the first, and taprostene [13], U-68215 [14], RS-93427 [15] for the second. Some prostaglandins substituted at the $C_{16}$ carbon atom with cycloalkyl, aryl, heterocyclyl [16], furyl [17], methylene-2-(2-thiophene) [18] or $\mathrm{C}_{15}$ with 2-indanyl [19], aryl or heteroaryl scafolds [20] are claimed in patents.

In this direction, we introduced bicyclo[3.3.0]octene or bicylo[3.3.0]octane fragments in the $\beta$-ketophosphonates used to build the $\omega$-side chain in a selective $E$-Horner-Wadsworth -Emmons- (E-HWE) reaction to obtain two PG analogs of types I and II (Figure 1): 

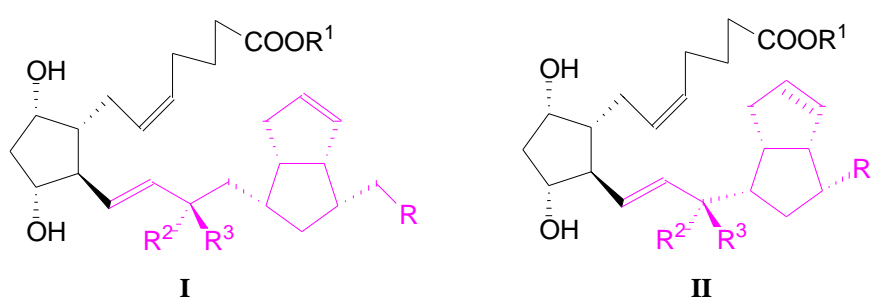

Figure 1. Prostaglandin analogs of type I with a bicyclo[3.3.0]octene fragment and respectively of type II with bicyclo[3.3.0]octene and bicyclo[3.3.0]octane fragments in the $\omega$-side chain.

In the first type, $\mathbf{I}$, the bicyclo[3.3.0]octene fragment is linked to the $\mathrm{C}_{16}$ carbon atom. This spacing from the $C_{15}$ carbon atom by a methylene group introduces a small, but significant hindrance of the 15-PGDH enzyme to inactivate the PG analogue by oxidation of $15 \alpha-\mathrm{OH}$ to the 15-keto group and keep the expected beneficial effect of the new bicyclo[3.3.0]octene fragment on the biological activity of the new PG analogs [21]. In the second type, II, the bulky bicyclo[3.3.0]octene and bicyclo[3.3.0]octane fragments, linked to the $\mathrm{C}_{15}$ carbon atom are expected to further slow down the inactivation of the PG analog by the oxidation of $15 \alpha-\mathrm{OH}$ to the 15-keto group via the 15-PGDH pathway [22]. Regarding both types I and II, we hope that the newly introduced bicyclo[3.3.0]oct(a)ene fragments will have a beneficial effect on the biological activity of the new PG analogs.

In this paper, we present the synthesis of new $\beta$-ketophosphonates, 5 , with a more bulky pentalenofurane fragment, linked to the ketone group, to build the PG analogs of type III (Figure 2):

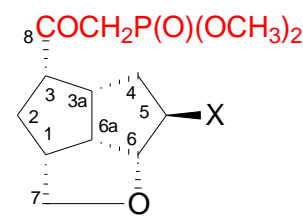

5. $\mathrm{X}=\mathrm{H}, \mathrm{I}, \mathrm{Br}$

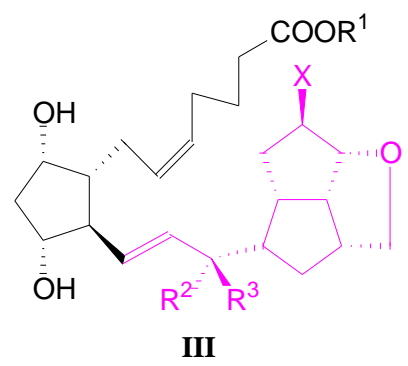

Figure 2. $\beta$-Ketophosphonates 5 (left) with a pentalenofurane fragment in the molecule, for obtaining new prostaglandin analogues of type III (right).

The biological activity of the PG analogs of type I-III and 8-10 was another goal we had; the synthesis of the corresponding $\beta$-ketophosphonates (used to build their $\omega$-side chain) was only the first step for obtaining these PG analogs. As we mentioned in the previous papers [21,22], a bicyclo[3.3.0]octa(e)ne fragment is encountered in many natural products, some with high anticancer activity. As far as we know, there is no mention in the literature for a bicyclo[3.3.0]octane (octahydropentalene) or bicyclo[3.3.0]octene (hexahydropentalene) fragment being introduced in $\alpha$ - or $\omega$-side chain of a PG analog. A pentalenofuran scaffold, a rigidized bicyclo[3.3.0]octane fragment with a tetrahydrofuran ring, was also never mentioned to be introduced in a PG analog. Some anticancer activity is expected to be found in the PG analogs (of type I-III and 8-10) with these scaffolds in the molecule, and we will follow up on it in the next papers.

\section{Results and Discussions}

For the synthesis of new $\beta$-ketophosphonates, the key step was the reaction of an ester with the lithium salt of dimethyl methanephosphonate, like in the previous papers [21-23]; the $\beta$-ketophosphonates are key intermediates for building the $\omega$-side chain in the total stereocontrolled convergent synthesis of prostaglandins and we have also used them for obtaining the intermediates used further in the hydrogenation of the double bond of the $\omega$-side chain [24]. 
The synthesis of the $\beta$-ketophosphonates, presented in Scheme 1 , started from the pentalenofurane alcohols 2 , previously obtained from regioselective reactions of the diol $\mathbf{1}$ [25]: by oxymercuration-demercuration reaction (compound $\mathbf{2 a}$ ) and respectively by haloetherification (for compounds $\mathbf{2 b}$ and $2 \mathbf{c}$ ) [26].

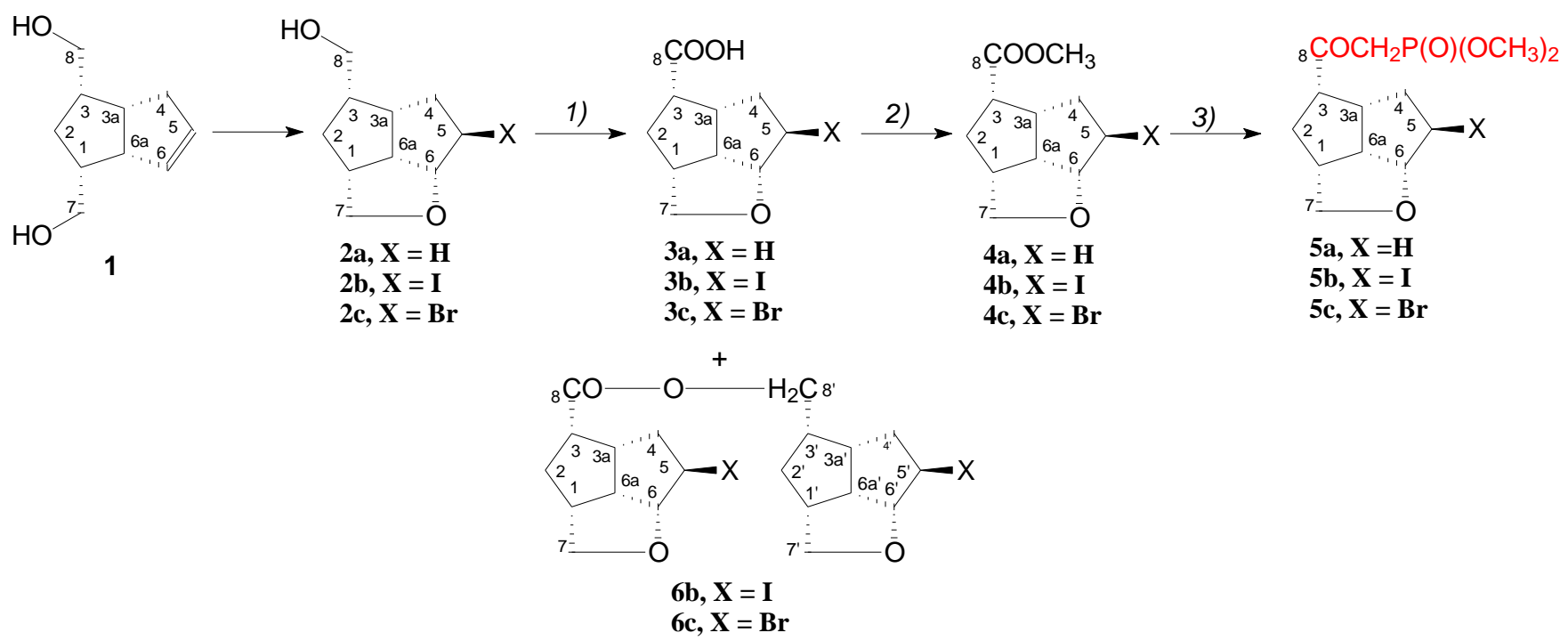

Scheme 1. Synthesis of pentalenofurane $\beta$-ketophosphonates 5a-5c. Reagents and Conditions: (1) $2.4 \mathrm{M}$ Jones reagent, acetone, -15 to $0{ }^{\circ} \mathrm{C}, 2 \mathrm{a}, 81.8 \%$ 3a; with $2 \mathbf{b}, 85.15 \%$ 3b; with $2 \mathbf{c}, 73.7 \%$ 3c, (2) MeOH, TsOH, rt, overnight, $86.4 \% 4 \mathbf{a} ; 92.4 \%$ $4 \mathbf{b} ; 81.0 \%$ 4c, (3) dimethyl methanephosphonate, $n$-BuLi, $-75{ }^{\circ} \mathrm{C}$ to $-65{ }^{\circ} \mathrm{C}, 88.0 \% 5 \mathbf{a} ; 78.6 \% 5 \mathbf{b} ; 83.3 \% 5 \mathbf{c}$.

Diols 2 were oxidized with $2.4 \mathrm{M}$ Jones reagent to afford acids 3 in good yields. In the reaction, the secondary compounds $6 b$ and $6 c$ resulted in small quantities. Their structure was established by NMR spectroscopy which showed that they had appeared by esterification of the acid resulted in the reaction, with the starting alcohols $\mathbf{2} \mathbf{b}$ and $\mathbf{2 c}$. This esterification is mentioned in the literature, for example in the reference [27]. A similar compound $\mathbf{6 a}$ resulted also in the synthesis of $\mathbf{3 a}$, but it was not isolated pure. Next, acids $\mathbf{2}$ were esterified in good yields with methanol in the presence of $\mathrm{TsOH}$ as acid catalyst. In the last step, esters $\mathbf{4 a - 4} \mathbf{c}$ were reacted with the lithium salt of dimethyl methanephosphonate at low temperature $\left(-75{ }^{\circ} \mathrm{C}\right.$ to $\left.-65^{\circ} \mathrm{C}\right)$ to give the $\beta$-ketophosphonates $\mathbf{5 a}-\mathbf{5 c}$, also in good yield.

The $\beta$-ketophosphonates $\mathbf{5 a - 5 c}$ will be used to obtain the PG analogs of type III (Figure 2) with the most steric hindrance to the $\mathrm{C}_{15 \alpha}-\mathrm{OH}$ group as compared to that encountered in compounds I and II (Figure 1); so, the inactivation of the PG analogs by oxidation of $15 \alpha-\mathrm{OH}$ to the 15-keto group via the 15-PGDH pathway is expected to be even more slowed.

We used the $\beta$-ketophosphonate $5 \mathrm{c}$ in the E-HWE selective olefination of the aldehyde 7, with the hydrogenated $\alpha$-side chain, for building the ketoprostaglandin analog 8 . As expected, the reduction of the enone group to the desired allylic alcohol 9 with the selective, but bulky reducing reagent aluminium diisobornyloxyisopropoxide, usually used in the PG field, did not proceed, as in the case of the PG analog II $\left(R^{1}, R^{2}=O\right)$ (Figure 1$)$. This finding strengthens our expectation that the inactivation of the PG analogs by oxidation of $15 \alpha-\mathrm{OH}$ to the 15-keto group via 15-PGDH pathway will be even more slowed than in the PG analogs of type II. The Luche reduction of the enone 8 with $\mathrm{NaBH}_{4}$ and $\mathrm{CeCl}_{3}$ gave the allylic alcohol 9 together with its 15-epimer, 10 (Scheme 2). So, we introduced for the first time a pentalenofurane scaffold in the $\omega$-side chain of a $F_{1} P G$ analog. These results will be presented separately [28]. 


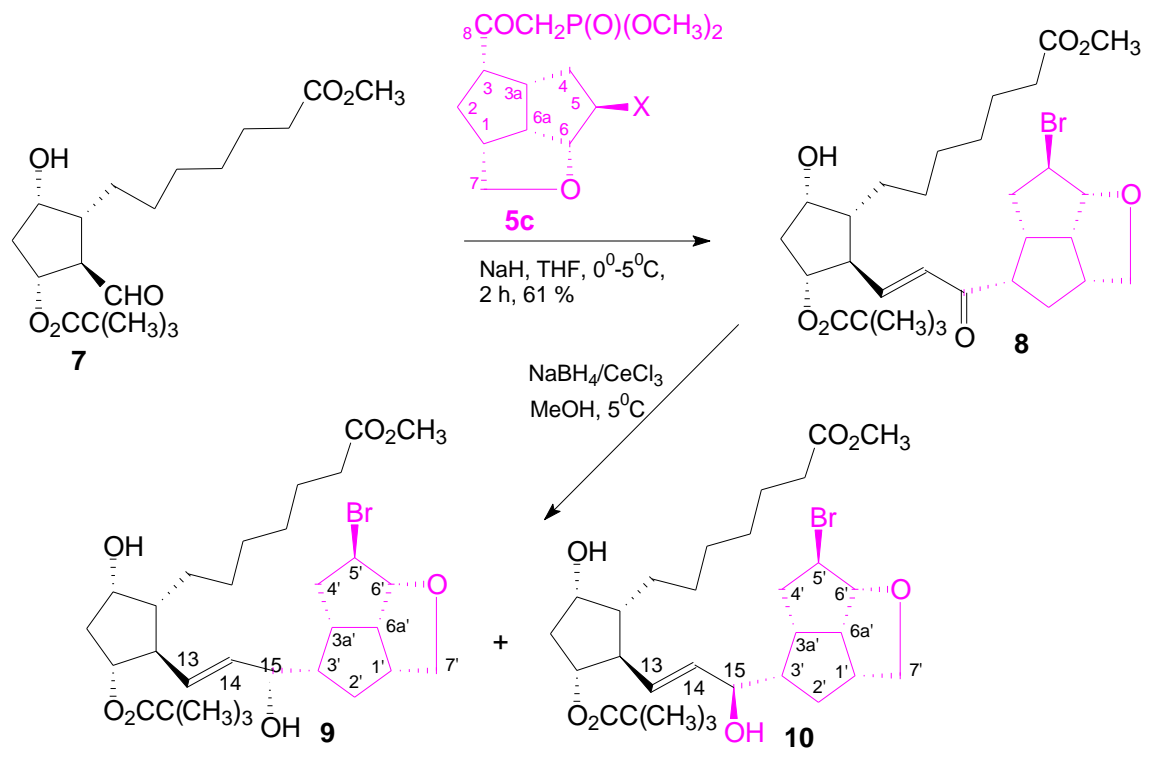

Scheme 2. Synthesis of $F_{1} P G$ analogs 8,9 and $\mathbf{1 0}$ with a pentalenofurane fragment in the $\omega$-side chain.

The ${ }^{1} \mathrm{H}$ - and ${ }^{13} \mathrm{C}-\mathrm{NMR}$ spectra are in agreement with the chemical structure of the compounds 2-6. The analysis of the proton spectra highlights several features. All methylene groups (positions 2, 4, 7 and respectively $2^{\prime}, 4^{\prime}$ and $8^{\prime}$ ) appear as $\mathrm{AB}$ systems but are highlighted as such only in the case of protons 7,8 and $7^{\prime}$ and $8^{\prime}$. In all the other cases, the vicinal and remote couplings cause the $\mathrm{AB}$ system not to appear explicitly. The solving of the $\mathrm{AB}$ systems was done with the help of two-dimensional homonuclear and heteronuclear correlation spectra.

In the $\beta$-ketophosphonate compounds $\mathbf{5 a}-\mathbf{5 c}$, the $\mathrm{H}-8$ protons, which form an $\mathrm{AB}$ system with a coupling constant of about $14 \mathrm{~Hz}$, are also coupled to phosphorus with a coupling constant of $22.7-22.9 \mathrm{~Hz}$. The presence of halogen in position 5 does not influence the chemical shift of $\mathrm{H}-5$, but in the case of the carbon spectra of compound $\mathbf{5 b}$ the strong iodine-induced shielding of C-5 is noticed, the chemical shift is lowered to $\delta=32.54 \mathrm{ppm}$. $\beta$-Ketophosphonates $\mathbf{5} \mathbf{a}, \mathbf{5} \mathbf{b}$, and $\mathbf{5} \mathbf{c}$ show couplings between the phosphorus atom and the methylene carbon $(J=128.5 \mathrm{~Hz})$, respectively the $\mathrm{C}-8$ carbonyl group $(J=6.2 \mathrm{~Hz})$. The methoxy group has couplings with phosphorus $\left(J_{(\mathrm{H} 8-\mathrm{P})}=11.2 \mathrm{~Hz}\right)$ and carbon $\left(J_{(\mathrm{C} 8-\mathrm{P})}=16.7 \mathrm{~Hz}\right)$.

The synthesis of the new $\beta$-ketophosphonates $\mathbf{5 a}-\mathbf{5} \mathbf{c}$ with a pentalenofuran scaffold linked to the ketone group started from the pentalenofuran alcohols $\mathbf{2 a}-\mathbf{2} \mathbf{c}$, whose chemical structure was confirmed by X-ray crystallography [26]. Chemical transformations of the exocyclic hydroxymethyl group from 2 to 5 in waiting to keep unmodified the pentalenofuran skeleton, but we tried to confirm their structure by X-ray crystallography. Though the $\beta$-ketophosphonates $\mathbf{5 b}$ and $\mathbf{5} \mathbf{c}$ were obtained crystallized as white beads and as puffy aces, the crystals were not suitable for single crystal determination of their structure, and not by XRPD powder diffraction method. The structure of the secondary compounds $\mathbf{6 b}$ and $\mathbf{6 c}$ was confirmed by X-ray crystallography, as follows:

- Compound $6 c$ provided suitable crystals for single crystal X-ray determination and its structural configuration is presented in Figure 3. As we observed in NMR spectra, the molecule $\mathbf{6} \mathbf{c}$ is an ester of the acid $\mathbf{3 c}$, formed in Jones oxidation of the alcohol $\mathbf{2 c}$, with the starting un-oxidized alcohol $\mathbf{2} \mathbf{c}$, presented in the reaction mixture until its complete oxidation. 


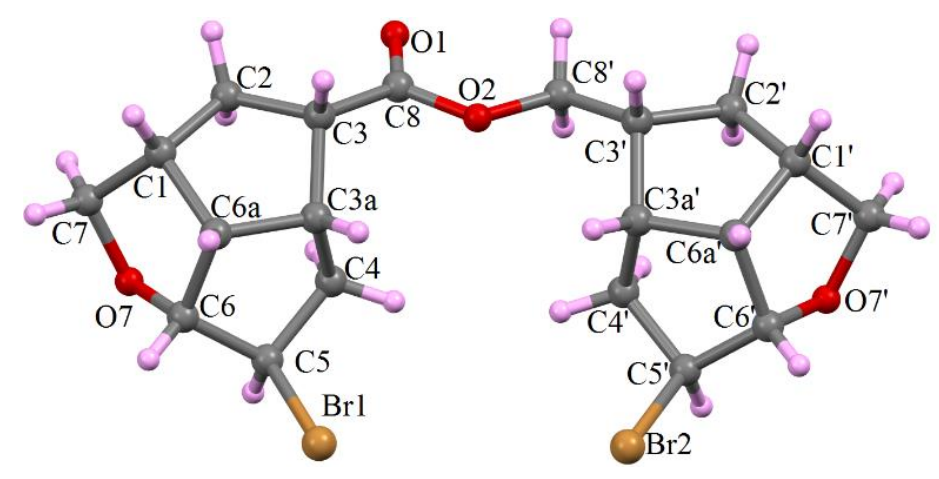

Figure 3. X-Ray molecular configuration of the asymmetric unit for secondary compound $\mathbf{6 c}$.

- $\quad$ By contrast with compound $\mathbf{6 c}$, compound $\mathbf{6 b}$ had no suitable single crystals and the structure was determined by the XRPD method, presented in detail at Experimental Section 3.2.

The experimental data results conducted to the structure $\mathbf{6 b}$, presented in Figure 4:

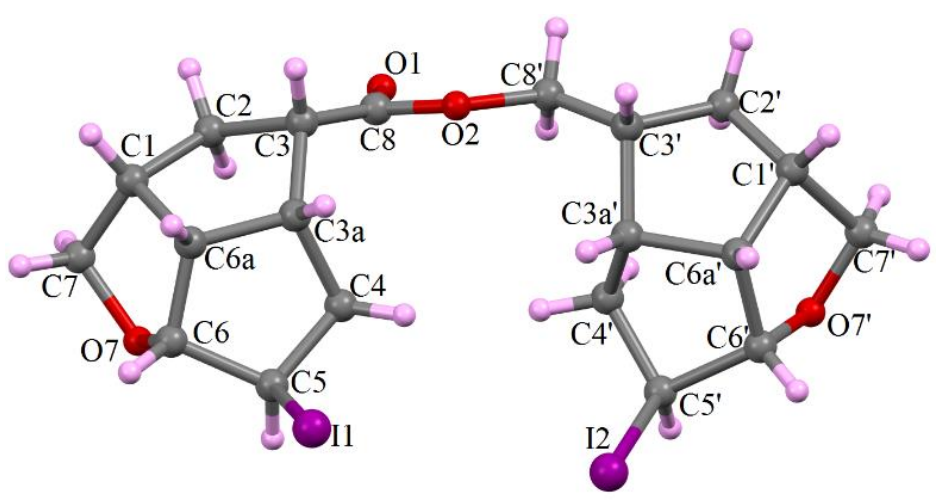

Figure 4. X-Ray molecular configuration of the asymmetric unit for secondary compound $\mathbf{6 b}$.

Detailed experimental procedures are presented in Experimental Section 3.2. X-ray crystallography. The main crystallographic data, bond lengths $(\AA)$ and angles $\left(^{\circ}\right)$ are given in Tables S2 and S3 (ESI), and the values are in agreement with the pentalenofuran structure of both moieties of the molecules, as previously presented for pentalenofuran alcohols 2 [26].

\section{Experimental}

\subsection{General Information}

Melting points (uncorrected) were determined in open capillary on an OpiMelt melting point apparatus (MPA 100, Stanford Research System, Inc., Sunnyvale, CA, USA). The progress of the reactions was monitored by TLC on silica gel 60 or $60 \mathrm{~F}_{254}$ plates (Merck, Darmstadt, Germany) eluted with the solvent systems: I, ethyl acetate-hexane-acetic acid, 5:1:0.1, II, ethyl acetate-hexane-acetic acid, 5:4:0.1. Spots were developed in UV and with $15 \% \mathrm{H}_{2} \mathrm{SO}_{4}$ in $\mathrm{MeOH}$ (heating at $110{ }^{\circ} \mathrm{C}, 10 \mathrm{~min}$ ). IR spectra were recorded on a FT-IR spectrometer (Bruker Vertex 70, Etilingen, Germany) by ATR and frequencies were expressed in $\mathrm{cm}^{-1}$, with the following abbreviations: $\mathrm{w}=$ weak, $\mathrm{m}=$ medium, $\mathrm{s}=$ strong, $\mathrm{v}=$ very, $\mathrm{br}=$ broad. HRMS spectra were recorded on LTQ Orbitrap XL (Thermo Fisher Scientific, Waltham, MA, USA). ${ }^{1} \mathrm{H}-\mathrm{NMR}$ and ${ }^{13} \mathrm{C}-\mathrm{NMR}$ spectra were recorded on a $300 \mathrm{MHz}$ spectrometer (Bruker, Rheinstatten, Germany) chemical shifts are given in ppm relative to TMS as internal standard. Complementary spectra: 2D-NMR and decoupling were done for the correct assignment of NMR signals. The numbering of the atoms in the compounds is presented in Scheme 1. Compound 2a was obtained by oxymercuration- 
demercuration reaction of the starting diol 1 [24], as we presented in a previous paper [25], and compounds $\mathbf{2} \mathbf{b}-\mathbf{2} \mathbf{c}$ were obtained by haloetherification of the same diol $\mathbf{1}$ [26].

\subsubsection{Chemistry: Synthesis of Compound 3a}

To a solution of compound $2 \mathrm{a}(18.09 \mathrm{~g}, 0.1075 \mathrm{~mol})$ in acetone $(500 \mathrm{~mL})$, cooled to $<-10{ }^{\circ} \mathrm{C}$ on an ice-salt bath, $2.4 \mathrm{M}$ Jones reagent $(85 \mathrm{~mL})$ was added dropwise during $1.5 \mathrm{~h}$, under energic mechanical stirring, maintaining the temperature below $0{ }^{\circ} \mathrm{C}$. The stirring was continued for $1 \mathrm{~h}$, monitoring the end of the reaction by TLC $\left(I, R_{f 2 a}=0.59, R_{f 3 a}=0.76\right)$. Isopropyl alcohol $(21 \mathrm{~mL})$ was added, the reaction mixture was stirred for $30 \mathrm{~min}$, the salts were filtered off [the salts were washed with $300 \mathrm{~mL}$ acetone, then dissolved in brine and extracted with ethyl acetate $(2 \times 150 \mathrm{~mL})]$ and the acetone solutions were concentrated under reduced pressure. The concentrate was taken into the unified ethyl acetate extracts, the solution was washed with brine $(50 \mathrm{~mL})$, dried $\left(\mathrm{MgSO}_{4}\right)$, concentrated to a crystallized mass (18.3 g) which was recrystallized from acetone-hexane; $10.53 \mathrm{~g}$ of pure $(2 \mathrm{a} S, 2 \mathrm{a} 1 S, 4 \mathrm{a} R, 5 S, 6 \mathrm{a} R)$-octahydro- $1 H$-pentaleno[1,6-bc]furan-5-carboxylic acid (3a) were obtained, $\mathrm{mp} 98.5-100.5^{\circ} \mathrm{C}$, FT-IR: 3375-2350 (large band, $\left(v_{\mathrm{OH}}\right), 2951 \mathrm{vs}\left(v_{\mathrm{CH} 2}\right.$ asymm. $)$, $2868 \mathrm{vs}\left(v_{\mathrm{CH} 2}\right.$ symm. $), 1687\left(v_{\mathrm{C}=\mathrm{O}}\right), 1460 \mathrm{~s}, 1425\left(\delta_{\mathrm{CH} 2}\right), 1355 \mathrm{~m}, 1290 \mathrm{~s}\left(v_{\mathrm{C}-\mathrm{O}-\mathrm{C}}\right), 1265 \mathrm{~s}, 1211 \mathrm{~m}$, $1105 \mathrm{~m}, 1075 \mathrm{~m}\left(v_{\mathrm{C}-\mathrm{OH}}\right), 1000 \mathrm{~m}, 896 \mathrm{~m}\left(v_{\mathrm{C}-\mathrm{O}-\mathrm{C}}\right), 801 \mathrm{~m}\left(v_{\mathrm{C}-\mathrm{O}-\mathrm{C}}\right), 717 \mathrm{~m}, 679 \mathrm{~m},{ }^{1} \mathrm{H}-\mathrm{NMR}\left(\mathrm{CDCl}_{3}\right.$, $\delta$ ppm, J Hz): 4.43 (dd, $1 \mathrm{H}, \mathrm{H}-6,3.2,6.0), 3.74(\mathrm{~d}, 1 \mathrm{H}, \mathrm{H}-7,8.9), 3.50$ (dd, $1 \mathrm{H}, \mathrm{H}-7,4.9,8.9)$, 2.98 (dt, 1H, H-6a, 6.0, 9.0), 2.88 (dq, 1H, H-3a, 6.6, 12.8), 2.73 (q, 1H, H-3, 8.0), 2.61 (dq, 1H, $\mathrm{H}-1,4.9,9.0), 2.06$ (dt, 1H, H-4, 7.4, 12.8), 1.99 (m, 1H, H-2), 1.75 (dt, 1H, H-4, 10.4, 12.8), 1.67 (m, 1H, H-5), 1.54-1.40 (m, 2H, H-2, H-5), ${ }^{13} \mathrm{C}-\mathrm{NMR}\left(\mathrm{CDCl}_{3}, \delta \mathrm{ppm}\right): 179.24$ (C-8), 85.43 (C-6), 73.25 (C-7), 54.57 (C-6a), 48.63 (C-3a), 46.46 (C-3), 44.03 (C-1), 33.46 (C-2), 32.39 (C-4), 25.99 (C-5), HRMS ESI (+) calc. for M+H, 183.1015, found [M + H] 183.1010. By LPC of the mother liquors (eluent: benzene-ethyl acetate, 2:1), another $3.32 \mathrm{~g}$ (total yield $81.8 \%$ ) of pure compound $3 \mathbf{a}$ were obtained and $2.48 \mathrm{~g}$ of pure starting alcohol $2 \mathrm{a}$ were recovered.

\subsubsection{Synthesis of Compound $\mathbf{3 b}$}

The iodo-pentalenofurane compound $\mathbf{2 b}(9.76 \mathrm{~g}, 33.18 \mathrm{mmol})$ in acetone $(175 \mathrm{~mL})$ was oxidized under the same conditions, presented above, with $2.4 \mathrm{M}$ Jones reagent $(29 \mathrm{~mL})$, monitoring the end of the reaction by TLC (II, $R_{f 2 b}=0.48, R_{f 3 b}=0.63$. A secondary compound was formed in the reaction with $\left.R_{f} 6 b=0.75\right)$. After the addition of isopropanol $(10 \mathrm{~mL})$ and work-up as previously, the concentrate was dissolved in ethyl acetate extracts $(2 \times 150 \mathrm{~mL})$, the resulting solution was washed with brine $(60 \mathrm{~mL}), 10 \% \mathrm{KHCO}_{3}$ solution $(3 \times 30 \mathrm{~mL})$, brine $(50 \mathrm{~mL})$, dried $\left(\mathrm{MgSO}_{4}\right)$ and concentrated under reduced pressure, afording 2.8 of the neutral secondary compound ( $2 \mathrm{a} R, 2 \mathrm{a} 1 S, 3 R, 4 \mathrm{aS}, 5 \mathrm{~S}, 6 \mathrm{a} R)-3$-iodooctahydro- $1 \mathrm{H}-$ pentaleno[1,6-bc]furan-5-yl)methyl ( $2 \mathrm{a} R, 2 \mathrm{a} 1 S, 3 R, 4 \mathrm{a} R, 5 \mathrm{~S}, 6 \mathrm{a} R)$-3-iodooctahydro-1H-pentaleno[1,6bc]furan-5-carboxylate $(\mathbf{6 b})$, which crystallized $(720 \mathrm{mg})$ from ethyl acetate-hexane, FT-IR: $2933 \mathrm{vs}\left(v_{\mathrm{CH} 2}\right.$ asymm. $), 2840 \mathrm{vs}\left(v_{\mathrm{CH} 2}\right.$ symm. $), 1700\left(v_{\mathrm{C}=\mathrm{O}}\right), 1447 \mathrm{~m}, 1338\left(\delta_{\mathrm{CH} 2}\right), 1271 \mathrm{~s}\left(v_{\mathrm{C}-\mathrm{O}-\mathrm{C}}\right)$, $1224 \mathrm{~m}, 1152 \mathrm{~s}, 1153 \mathrm{~s}, 992 \mathrm{~s}, 936 \mathrm{~m}\left(v_{\mathrm{C}-\mathrm{C}}\right), 908 \mathrm{~m}\left(v_{\mathrm{C}-\mathrm{O}-\mathrm{C}}\right), 809 \mathrm{~m}\left(\mathrm{v}_{\mathrm{C}-\mathrm{O}-\mathrm{C}}\right),{ }^{1} \mathrm{H}-\mathrm{NMR}\left(\mathrm{CDCl}_{3}, \delta\right.$ ppm, J Hz): 4.56 (d, 1H, H-6, 5.1), 4.55 (d, 1H, H-6', 5.1), 4.43 (d, 2H, H-5, H-5' , 5.1), 4.04 (dd, $\left.1 \mathrm{H}, \mathrm{H}-8^{\prime}, 6.6,11.2\right), 3.94$ (dd, $\left.1 \mathrm{H}, \mathrm{H}-8^{\prime}, 8.4,11.2\right), 3.81(\mathrm{~d}, 1 \mathrm{H}, \mathrm{H}-7$ or H-7', 8.9), 3.77 (d, $1 \mathrm{H}, \mathrm{H}-7$ or $\left.\mathrm{H}-7^{\prime}, 8.9\right)$, 3.56 (d, $1 \mathrm{H}, \mathrm{H}-7$ or H-7', 8.9$), 3.53$ (d, $1 \mathrm{H}, \mathrm{H}-7$ or H-7', 8.9), 3.36-3.19 (m, 3H, H-3, H-6a, H-6' a) , 3.02-2.95 (m, 2H, H-1, H-1'), 2.76-2.62 (m, 2H, H-3a, H-3'a), 2.38 (m, 1H, H-3'), 2.25-1.96 (m, 6H, 2H-4, 2H-4' , H-2, H-2'),1.88 (dd, 1H, H-4' $\left.{ }^{\prime}, 10.2,14.6\right), 1.72$ (dt, 1H, H-2, 9.7, 13.1), ${ }^{13} \mathrm{C}-\mathrm{NMR}\left(\mathrm{CDCl}_{3}, \delta\right.$ ppm): 172.77 (COO), 92.69, 92.37 (C-6, C-6'), 74.32, 74.15 (C-7, C-7'), 65.15 (C-8'), 53.41, 53.29 (C-6a, C-6a'), 46.04 (C-3), 42.31 (C-3'), 47.59 (C-3a), $46.01\left(\mathrm{C}-3 \mathrm{a}^{\prime}\right), 43.37$ (C-1), $42.31\left(\mathrm{C}-1^{\prime}\right), 37.12\left(\mathrm{C}-4^{\prime}\right), 35.33$ (C-4), $34.59\left(\mathrm{C}-2^{\prime}\right), 33.23$ (C-2), 33.18, 32.76 (C-5, C-5'), HRMS calc. M + H: 587.0149, found: 587.2804.

The $\mathrm{KHCO}_{3}$ solutions were acidified to $\mathrm{pH} 2$ with $2 \mathrm{~N} \mathrm{HCl}$, the resulting precipitate was extracted with ethyl acetate $(3 \times 75 \mathrm{~mL})$, the extracts were washed with brine $(30 \mathrm{~mL})$, dried $\left(\mathrm{Na}_{2} \mathrm{SO}_{4}\right)$, concentrated and the crude product was crystallized from ethyl acetate-hexane, resulting in $8.5 \mathrm{~g}(85.15 \%)$ of $(2 \mathrm{a} R, 2 \mathrm{a} 1 \mathrm{~S}, 3 R, 4 \mathrm{a} R, 5 \mathrm{~S}, 6 \mathrm{a} R)$-3-iodooctahydro-1H-pentaleno[1,6bc]furan-5-carboxylic acid (3b), as prisms, mp 166.0-169.0 ${ }^{\circ} \mathrm{C}$ (dec), IR: 2959m, 2851m, 
2641br, 1685vs, 1419m, 1249m, 1109m, 1058s, 906s, 593w, ${ }^{1} \mathrm{H}-\mathrm{NMR}\left(\mathrm{CDCl}_{3}, \delta \mathrm{ppm}, J \mathrm{~Hz}\right)$ : 4.56 (d, 1H, H-6, 5.7), 4.42 (d, 1H, H-5, 3.0), 3.80 (d, 1H, H-7, 8.9), 3.54 (dd, 1H. H-7, 5.0, 8.9), 3.36-3.19 (m, 2H, H-6a, H-3a), 2.98 (dt, 1H, H-3, 7.0, 10.2), 2.68 (dq, 1H, H-1, 5.0, 9.4), 2.21 (dd, 1H, H-2, 7.4, 13.0), 2.12 (m, 2H, H-4), 1.73 (dd, 1H, H-2, 10.2, 13.0), ${ }^{13} \mathrm{C}-\mathrm{NMR}$ $\left(\mathrm{CDCl}_{3}, \delta \mathrm{ppm}\right.$ ): 179.02 (COO), 92.76 (C-6), 74.11 (C-7), 53.35 (C-6a), 47.41 (C-3), 45.93 (C-3a), 43.40 (C-1), 37.03 (C-4), 33.47 (C-2), 32.45 (C-5), HRMS ESI (+) calc. for $\mathrm{M}+\mathrm{H}$, 308.9982, found 309.1297.

\subsubsection{Synthesis of Compound 3c}

Bromoalcohol 2c $(11.67 \mathrm{~g}, 47 \mathrm{mmol})$, dissolved in acetone $(250 \mathrm{~mL})$, was oxidized as in example 1: $2.4 \mathrm{M}$ Jones reagent $(37 \mathrm{~mL})$, isopropyl alcohol $(16 \mathrm{~mL}), \mathrm{TLC}\left(\mathrm{II}, \mathrm{R}_{\mathrm{f} 2 \mathrm{c}}=0.43\right.$, $\left.R_{f 3 c}=0.57, R_{f 6 c}=0.72\right)$. The work-up of the reaction as in example 2 gave $9.04 \mathrm{~g}(73.7 \%)$ of crystallized acid ( $2 \mathrm{a} R, 2 \mathrm{a} 1 S, 3 R, 4 \mathrm{a} R, 5 \mathrm{~S}, 6 \mathrm{aR})$-3-bromooctahydro- $1 H$-pentaleno[1,6-bc]furan5-carboxylic acid (3c), mp 159-160.0 ${ }^{\circ} \mathrm{C}$, IR: 2964m, 2863m, 2684br, 1690vs, $1430 \mathrm{~m}, 1213 \mathrm{~m}$, 1065m, 910m, 620w, ${ }^{1} \mathrm{H}-\mathrm{NMR}\left(\mathrm{CDCl}_{3}, \delta \mathrm{ppm}, J \mathrm{~Hz}\right): 4.40$ (d, 1H, H-5, 5.0), 3.78 (d, 1H, H-7, 8.9), 3.56 (dd, $1 \mathrm{H}, \mathrm{H}-7,5.0,8.9), 3.28$ (m, 1H, H-6a), 3.22 (m, 1H, H-3a), 2.97 (dt, 1H, H-3, 6.5, 12.8), 2.70 (dt, 1H, H-1, 5.0, 9.4), 2.18 (m, 1H, H-2), 2.14 (m, 2H, H-4), 1.73 (td, 1H, H-2, 10.2, 13.0), ${ }^{13} \mathrm{C}-\mathrm{NMR}\left(\mathrm{CDCl}_{3}, \delta \mathrm{ppm}\right): 179.01$ (COO), 90.90 (C-6), 74.08 (C-7), 55.20 (C-5), 53.29 (C-6a), 47.63 (C-3), 45.10 (C-3a), 43.36 (C-1), 35.63 (C-4), 33.17 (C-2), HRMS ESI (+) calc. for $\mathrm{M}+\mathrm{H}, 261.0120 / 263.0100$, found 261.1088/263.0880.

By LPC purification of the ethyl acetate phases extracted with $10 \% \mathrm{KHCO}_{3}$ soln., $1.20 \mathrm{~g}(5.5 \%)$ of pure $(2 \mathrm{a} R, 2 \mathrm{a} 1 S, 3 R, 4 \mathrm{a} S, 5 S, 6 \mathrm{a} R)$-3-bromooctahydro- $1 H$-pentaleno[1,6-bc]furan5-yl)methyl $\quad(2 \mathrm{a} R, 2 \mathrm{a} 1 S, 3 R, 4 \mathrm{a} R, 5 S, 6 \mathrm{a} R)-3$-bromooctahydro-1H-pentaleno[1,6-bc]furan-5carboxylate (6c), resulted, $\mathrm{mp} 122.0-124.0{ }^{\circ} \mathrm{C}$, IR: 2927s, 2852s, $1711 \mathrm{vs}, 1460 \mathrm{w}, 1178 \mathrm{vs}$, 1060s, 618m, ${ }^{1} \mathrm{H}-\mathrm{NMR}\left(\mathrm{CDCl}_{3}, \delta \mathrm{ppm}, J \mathrm{~Hz}\right): 4.46-4.37$ (m, 4H, H-5, H-6 $\left.{ }^{\prime}, \mathrm{H}-5^{\prime}, \mathrm{H}-6\right)$, 4.10-3.90 (m, 2H, H-8'), 3.84-3.53 (m, 4H, 2H-7, 2H-7'), 3.35-3.15 (m, 2H, H-6a, H-6a'), 3.02-2.89 (m, 2H, H-3a, H-3a'), 2.80-2.65 (m, 2H, H-1, H-1'), 2.39 (H-3), 2.23-1.65 (m, 8H, $\left.\mathrm{H}-3^{\prime}, 2 \mathrm{H}-2, \mathrm{H}-2^{\prime}, 2 \mathrm{H}-4,2 \mathrm{H}-4^{\prime}\right), 1.17$ (dt, $\left.1 \mathrm{H}, \mathrm{H}-2^{\prime}, 9.9,12.7\right),{ }^{13} \mathrm{C}-\mathrm{NMR}\left(\mathrm{CDCl}_{3}, \delta \mathrm{ppm}\right)$ : 172.85 (COO), 90.93, 90.64 (C-6, C-6' $), 74.36,74.19$ (C-7, C-7'), 65.15 (C-8') , 55.78, 55.45 (C-5, C-5') , 53.47, 53.35 (C-6a, C-6a'), 47.89, 45.30 (C-3. C-3'), 44.64, 43.92 (C-3a, C-3a'), $43.45,42.64\left(\mathrm{C}-1, \mathrm{C}-1^{\prime}\right), 35.80,34.38\left(\mathrm{C}-4, \mathrm{C}-4^{\prime}\right), 34.05,33.46$ (C-2, C-2'), HRMS calc. M + H: 489.0271/491.0250/493.0230, found: 489.0396/491.0232/493.0210.

\subsubsection{Synthesis of Compound 4a}

Compound 3a ( $5.26 \mathrm{~g}, 28.86 \mathrm{mmol})$ was dissolved in methanol $(175 \mathrm{~mL}), \mathrm{TsOH}(200 \mathrm{mg})$ was added and the solution was stirred overnight at $\mathrm{rt}$, monitoring the end of the reaction by TLC (II, $\left.\mathrm{R}_{\mathrm{f} 3 \mathrm{a}}=0.55, \mathrm{R}_{\mathrm{f} 4 \mathrm{a}}=0.67\right)$. $\mathrm{KHCO}_{3}(0.5 \mathrm{~g})$ was added, the solution was stirred for $30 \mathrm{~min}$, the solvent was distilled under reduced pressure, the residue was taken in water $(20 \mathrm{~mL})$ and chloroform $(150 \mathrm{~mL})$, the phases were separated, organic phase was washed with sat. soln. $\mathrm{NaHCO}_{3}(30 \mathrm{~mL})$ (aqueous phases extracted with more $30 \mathrm{~mL}$ chloroform), brine $(30 \mathrm{~mL})$, dried $\left(\mathrm{Na}_{2} \mathrm{SO}_{4}\right)$, concentrated and the crude product was purified by LPC (ethyl acetate-hexanes, 1:1). A pure fraction of methyl (2aS,2a1S,4aR,5S,6aR)-octahydro- $1 \mathrm{H}$ pentaleno[1,6-bc]furan-5-carboxylate (4a, $4.89 \mathrm{~g}, 86.4 \%)$ was obtained as an oil, IR: 2909vs $\left(v_{\mathrm{CH} 2}\right.$ asymm. $), 2833 \mathrm{vs}\left(v_{\mathrm{CH} 2}\right.$ symm. $), 1713 \mathrm{vs}\left(v_{\mathrm{C}}=\mathrm{O}\right), 1492\left(\delta_{\mathrm{CH} 2}\right), 1347 \mathrm{~m}, 1256 \mathrm{~s}, 982 \mathrm{~s}, 882 \mathrm{~m}$, ${ }^{1} \mathrm{H}-\mathrm{NMR}\left(\mathrm{CDCl}_{3}, \delta \mathrm{ppm}, J \mathrm{~Hz}\right): 4.21(\mathrm{dd}, 1 \mathrm{H}, \mathrm{H}-6,3.4,6.1), 3.73$ (d, $\left.1 \mathrm{H}, \mathrm{H}-7,8.9\right), 3.65(\mathrm{~s}, 3 \mathrm{H}$, $\left.\mathrm{OCH}_{3}\right), 3.50$ (dd, 1H, H-7, 4.8, 8.9), 2.97 (dt, 1H, H-6a, 6.3, 9.3), 2.85 (dt, 1H, H-3a, 6.6, 12.8), 2.72 (m,1H, H-3), 2.61 (ddt, 1H, H-1, 4.8, 10.0, 12.9), 2.08 (m, 1H, H-4), 2.00 (m, 1H, H-2, 6.9, 12.9), 1.78 (dt, $1 \mathrm{H}, \mathrm{H}-4,10.2,12.9), 1.61-1.43(\mathrm{~m}, 2 \mathrm{H}, 2 \mathrm{H}-5), 1.45(\mathrm{~m}, 1 \mathrm{H}, \mathrm{H}-2),{ }^{13} \mathrm{C}-\mathrm{NMR}$ $\left(\mathrm{CDCl}_{3}, \delta \mathrm{ppm}\right): 173.90$ (COO), $85.40(\mathrm{C}-6), 73.38(\mathrm{C}-7), 54.58(\mathrm{C}-6 \mathrm{a}), 51.37\left(\mathrm{CH}_{3} \mathrm{O}\right), 48.72$ (C-3a), 46.64 (C-3), 44.14 (C-1), 33.55 (C-4), 32.69 (C-2), 26.03 (C-5), HRMS ESI (+) calc. for $\mathrm{M}+\mathrm{H}, 197.1172$, found 197.1165. 


\subsubsection{Synthesis of Compound $4 \mathrm{~b}$}

Compound $\mathbf{3 b}(5.06 \mathrm{~g}, 16.4 \mathrm{mmol})$ was esterified with methanol $(175 \mathrm{~mL})$ and $\mathrm{TsOH}$ $\left(200 \mathrm{mg}\right.$ ) as acid catalyst as in example 4 . TLC (II, $\left.R_{\mathrm{f} 3 \mathbf{b}}=0.48, R_{\mathrm{f} 4 \mathbf{b}}=0.63\right)$. The work-up as in example 4 gave $4.88 \mathrm{~g}(92.4 \%)$ of pure methyl ( $2 \mathrm{a} R, 2 \mathrm{a} 1 S, 3 R, 4 \mathrm{a} R, 5 S, 6 \mathrm{a} R)-3$-iodooctahydro$1 \mathrm{H}$-pentaleno[1,6-bc]furan-5-carboxylate $\left(4 \mathbf{b}\right.$, as long needles, mp $69.5-70.5^{\circ} \mathrm{C}$, IR: $2972 \mathrm{~s}$, $2945 \mathrm{vs}\left(v_{\mathrm{CH} 2}\right.$ asymm. $), 2847 \mathrm{vs}\left(v_{\mathrm{CH} 2}\right.$ symm. $), 1736 \mathrm{vs}\left(v_{\mathrm{C}=\mathrm{O}}\right), 1461 \mathrm{~s}, 1434 \mathrm{~s}\left(\delta_{\mathrm{CH} 2}\right), 1377 \mathrm{~s}, 1271 \mathrm{~m}$, $1171 \mathrm{~s}, 1070 \mathrm{~m}, 951 \mathrm{~s}, 914 \mathrm{~s}\left(v_{\mathrm{C}-\mathrm{O}-\mathrm{C}}\right), 810 \mathrm{~s}$ ( $\left.v_{\mathrm{C}-\mathrm{O}-\mathrm{C}}\right), 797 \mathrm{~s},{ }^{1} \mathrm{H}-\mathrm{NMR}\left(\mathrm{CDCl}_{3}, \delta \mathrm{ppm}, J \mathrm{~Hz}\right): 4.55$ (d, 1H, H-6, 5.8), 4.42 (d, $1 \mathrm{H}, \mathrm{H}-5,5.7), 3.80(\mathrm{~d}, 1 \mathrm{H}, \mathrm{H}-7,8.9), 3.66\left(\mathrm{~s}, 3 \mathrm{H}, \mathrm{CH}_{3} \mathrm{O}\right), 3.54(\mathrm{dd}$, $1 \mathrm{H}, \mathrm{H}-7,5.0,8.9), 3.30$ (dt, 1H, H-6a, 5.8, 8.8), 3.21 (dt, 1H, H-3a, 6.8, 9.5), 2.95 (dt,1H, H-3, 6.6, 12.8), 2.68 (ddt, $1 \mathrm{H}, \mathrm{H}-1,5.0,8.5,9.9), 2.20$ (dt 1H, H-2, 6.7, 13.0), 2.09 (ddt, $1 \mathrm{H}, \mathrm{H}-4$, $4.7,9.9,14.8), 1.99(\mathrm{dd}, 1 \mathrm{H}, \mathrm{H}-4,9.3,14.8), 1.76(\mathrm{dt}, 1 \mathrm{H}, \mathrm{H}-2,10.0,13.0),{ }^{13} \mathrm{C}-\mathrm{NMR}\left(\mathrm{CDCl}_{3}, \delta\right.$ ppm): 173.42 (COO), 92.74 (C-6), 74.16 (C-7), 53.29 (C-6a), $51.57\left(\mathrm{CH}_{3} \mathrm{O}\right), 47.41(\mathrm{C}-3), 46.03$ (C-3a), 43.37 (C-1), 37.04 (C-4), 33.72 (C-2), 32,82 (C-5), HRMS ESI (+) calc. for M + H, 323.0138, found 323.0128 .

\subsubsection{Synthesis of Compound $4 \mathrm{c}$}

Compound 3c (5.41 g, $20.7 \mathrm{mmol})$ was esterified as in example 4: methanol $(125 \mathrm{~mL})$, $\mathrm{TsOH}(150 \mathrm{mg}), 3$ days, $\mathrm{KHCO}_{3}(400 \mathrm{mg}), \mathrm{TLC}\left(\mathrm{II} \mathrm{R} \mathrm{R}_{\mathrm{f} z \mathrm{c}}=0.57, \mathrm{R}_{\mathrm{f} 4 \mathrm{c}}=0.75\right)$. The crude product was purified by LPC (eluent, ethyl acetate-hexanes, 1:2) and crystallized from ethyl acetate-hexane, resulting in $4.61 \mathrm{~g}(81.0 \%)$ of pure methyl $(2 \mathrm{a} R, 2 \mathrm{a} 1 S, 3 R, 4 \mathrm{a} R, 5 S, 6 \mathrm{a} R)-3-$ bromooctahydro- $1 \mathrm{H}$-pentaleno[1,6-bc]furan-5-carboxylate (4c), mp 57.5-58.0 ${ }^{\circ} \mathrm{C}$, IR: $2936 \mathrm{~s}$, 2842s, 1723vs, $1432 \mathrm{~m}, 1193 \mathrm{~m}, 1163 \mathrm{vs}, 801 \mathrm{~m}, 619 \mathrm{w},{ }^{1} \mathrm{H}-\mathrm{NMR}\left(\mathrm{CDCl}_{3}, \delta \mathrm{ppm}, J \mathrm{~Hz}\right): 4.40-4.39$ (m, 2H, H-5, H-6), 3.78 (d, 1H, H-7, 8.8), 3.66 (s, 3H, $\mathrm{CH}_{3} \mathrm{O}$ ), 3.56 (dd, $\left.1 \mathrm{H}, \mathrm{H}-7,5.0,8.8\right), 3.29$ (dt, 1H, H-6a, 6.0, 8.7), 3.19 (m, 1H, H-3a), 2.94 (dt,1H, H-3, 6.6, 12.9), 2.69 (dq, 1H, H-1, 5.0, 9.4), 2.21-2.09 (m, 2H, H-2, H-4), 2.01 (dd, $1 \mathrm{H}, \mathrm{H}-4,9.3,14.1), 1.75$ (dt, 1H, H-2, 10.0, 13.0), ${ }^{13} \mathrm{C}-\mathrm{NMR}\left(\mathrm{CDCl}_{3}, \delta \mathrm{ppm}\right): 173.41$ (COO), 90.93 (C-6), 74.17 (C-7), 55.47 (C-5), 53.27 (C-6a), $51.55\left(\mathrm{CH}_{3} \mathrm{O}\right), 47.68$ (C-3), 45.25 (C-3a), 43.39 (C-1), 35.64 (C-4), 33.42 (C-2), HRMS ESI (+) calc. for $\mathrm{M}+\mathrm{H}, 275.0277 / 277.0256$, found $275.0269 / 277.0248$.

\subsubsection{Synthesis of $\beta$-Ketophosphonate $5 \mathbf{a}$}

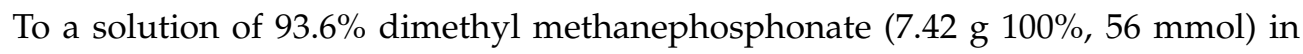
anhydrous tetrahydrofuran (THF) $(60 \mathrm{~mL})$, cooled to $-70{ }^{\circ} \mathrm{C}$ under an argon atmosphere using an ULTRA-KRYOMAT system (Lauda Dr. R. Wobser \& Co, Germany, a solution of $1.5 \mathrm{M} \mathrm{n}$-butyllitium $(39 \mathrm{~mL}$ ) in hexane was added dropwise under mechanical stirring, maintaining the reaction temperature below $-65^{\circ} \mathrm{C}$. The stirring was continued for $30 \mathrm{~min}$, a solution of the ester compound $4 \mathbf{a}(4.21 \mathrm{~g}, 21.45 \mathrm{mmol})$ in THF $(25 \mathrm{~mL})$ was added dropwise and the progress of the reaction was followed by TLC (II, $R_{f 4 a}=0.67$, $\left.R_{f 5 a}=0.16\right)$. Acetic acid $(3.7 \mathrm{~mL})$ was added, stirring was continued for $15 \mathrm{~min}$, the reaction mixture was transferred into a round bottom flask and concentrated under reduced pressure. The residue was taken in water $(70 \mathrm{~mL})$ and chloroform $(100 \mathrm{~mL})$, the phases were separated (aqueous phase was extracted with $2 \times 100 \mathrm{~mL}$ chloroform), the organic phase was washed with brine $(70 \mathrm{~mL})$, dried $\left(\mathrm{Na}_{2} \mathrm{SO}_{4}\right)$ and concentrated. The crude product $(11.7 \mathrm{~g})$ was purified by LPC (ethyl acetate-hexanes, 1:1), resulting in $4.10 \mathrm{~g}(88.0 \%)$ of the pure $\beta$-ketophosphonate dimethyl (2-((2aS,2a1S,4aR,5S,6aR)-octahydro- $1 H$-pentaleno[1,6bc]furan-5-yl)-2-oxoethyl)phosphonate (5a), as an oil, ${ }^{1} \mathrm{H}-\mathrm{NMR}\left(\mathrm{CDCl}_{3}, \delta \mathrm{ppm}, J \mathrm{~Hz}\right): 4.21$ (dd, $1 \mathrm{H}, \mathrm{H}-6,3.7,6.3), 3.80$ (d, 3H, $\left.\mathrm{CH}_{3} \mathrm{OP}, 11.3\right), 3.79$ (d, 3H, $\left.\mathrm{CH}_{3} \mathrm{OP}, 11.3\right), 3.73$ (d, 1H, H-7, 9.0), $3.51(\mathrm{dd}, 1 \mathrm{H}, \mathrm{H}-7,4.9,9.0), 3.26\left(\mathrm{dd}, 1 \mathrm{H}, \mathrm{CH}_{2} \mathrm{P}, J_{\mathrm{H}-\mathrm{P}}=22.7, J_{\text {vic }}=14.0\right), 3.258(\mathrm{dt}, 1 \mathrm{H}$, $\mathrm{H}-3,6.7,12.4), 3.05(\mathrm{dt}, 1 \mathrm{H}, \mathrm{H}-6 \mathrm{a}, 6.4,9.3), 3.01\left(\mathrm{dd}, 1 \mathrm{H}, \mathrm{CH}_{2} \mathrm{P}, J_{\mathrm{H}-\mathrm{P}}=22.7, J_{\text {vic }}=14.0\right), 2.79$ (dt, 1H, H-3a, 4.9, 10.2), 2.65 (ddt, 1H, H-1, 4.8, 8.5, 12.6), 1.99 (m, 2H, H-2, H-4), 1.79 (dt, $1 \mathrm{H}$, H-2, 10.2, 12.6), 1.50 (m, 1H, H-4), 1.45 (m,1H, H-5), 1.38 (dt, 1H, H-5, 3.7, 6.3), ${ }^{13}$ C-NMR $\left(\mathrm{CDCl}_{3}, \delta \mathrm{ppm}, J \mathrm{~Hz}\right): 201.58\left(\mathrm{CO}, J_{\mathrm{C}-\mathrm{P}}=6.2\right), 85.02(\mathrm{C}-6), 73.42$ (C-7), 57.79 (C-6a), 55.04 $(\mathrm{C}-3), 53.22\left(\mathrm{~d}, \mathrm{CH}_{3} \mathrm{OP}, J_{\mathrm{C}-\mathrm{P}}=6.7\right), 53.02\left(\mathrm{~d}, \mathrm{CH}_{3} \mathrm{OP}, J_{\mathrm{C}-\mathrm{P}}=6.7\right), 46.44(\mathrm{C}-3 \mathrm{a}), 44.01(\mathrm{C}-1)$, $40.50\left(\mathrm{~d}, \mathrm{CH}_{2} \mathrm{P}, J_{\mathrm{C}-\mathrm{P}}=129.4\right), 37.72(\mathrm{C}-4), 31.50(\mathrm{C}-2), 25.68(\mathrm{C}-5)$. 


\subsubsection{Synthesis of $\beta$-Ketophosphonate $\mathbf{5 b}$}

The $\beta$-ketophosphonate $\mathbf{5 b}$ was synthesized as in example 7: $93.6 \%$ dimethyl methanephosphonate $(4.66 \mathrm{~g} 100 \%, 37.6 \mathrm{mmol})$ in anhydrous THF $(35 \mathrm{~mL}), 1.5 \mathrm{M} n$-butyllitium $(24.5 \mathrm{~mL})$ in hexane, ester $4 \mathbf{b}(4.64 \mathrm{~g}, 14.4 \mathrm{mmol})$ in THF $(20 \mathrm{~mL})$, acetic acid $(2.5 \mathrm{~mL})$, TLC (II, $\left.R_{f 4 b}=0.77, R_{f 5 b}=0.18\right)$. The crude product was crystallized from ethyl acetate-hexane, resulting $4.69 \mathrm{~g}(78.6 \%)$ of pure $\beta$-ketophosphonate dimethyl (2-( $(2 \mathrm{a} R, 2 \mathrm{a} 1 S, 3 R, 4 \mathrm{a} R, 5 \mathrm{~S}, 6 \mathrm{a} R)$ 3-iodooctahydro- $1 H$-pentaleno[1,6-bc]furan-5-yl)-2-oxoethyl)phosphonate (5b), as white beads, mp $70.5-72.5^{\circ} \mathrm{C}$, IR: 2951s, $2850 \mathrm{~m}, 1696 \mathrm{~s}, 1460 \mathrm{~m}, 1245 \mathrm{~s}, 1167 \mathrm{~m}, 1024 \mathrm{vs}, 957 \mathrm{~m}, 806 \mathrm{~s}$, $547 \mathrm{w}, 505 \mathrm{~m},{ }^{1} \mathrm{H}-\mathrm{NMR}\left(\mathrm{CDCl}_{3}, \delta \mathrm{ppm}, J \mathrm{~Hz}\right): 4.49$ (d, $\left.1 \mathrm{H}, \mathrm{H}-6,5.7\right), 4.36(\mathrm{~d}, 1 \mathrm{H}, \mathrm{H}-5,4.5)$, $3.80\left(\mathrm{~d}, 3 \mathrm{H}, \mathrm{CH}_{3} \mathrm{OP}, 11.1\right), 3.79\left(\mathrm{~d}, 3 \mathrm{H}, \mathrm{CH}_{3} \mathrm{OP}, 11.1\right), 3.76(\mathrm{~d}, 1 \mathrm{H}, \mathrm{H}-7,8.9), 3.50(\mathrm{dd}, 1 \mathrm{H}$, H-7, 5.0, 8.9), 3.23-3.12 (m, 3H, H-6a, H-3a, H-3), 3.18 (dd, $1 \mathrm{H}, \mathrm{CH}_{2} \mathrm{P}, J_{\mathrm{H}-\mathrm{P}}=22.9, J_{\text {vic }}=13.7$ ), $2.97\left(\mathrm{dd}, 1 \mathrm{H}, \mathrm{CH}_{2} \mathrm{P}, J_{\mathrm{H}-\mathrm{P}}=22.9, J_{\text {vic }}=13.7\right), 2.67(\mathrm{dq}, 1 \mathrm{H}, \mathrm{H}-1,5.3,9.4), 2.06(\mathrm{dd}, 1 \mathrm{H}, \mathrm{H}-2$, 6.4, 10.4), 1.98-1.89 (m, 2H, H-4), 1.75 (dt, $1 \mathrm{H}, \mathrm{H}-2,10.4,13.8),{ }^{13} \mathrm{C}-\mathrm{NMR}\left(\mathrm{CDCl}_{3}, \delta \mathrm{ppm}\right)$ : $201.58\left(\mathrm{CO}, J_{\mathrm{C}-\mathrm{P}}=6.2\right), 92.39(\mathrm{C}-6), 74.05(\mathrm{C}-7), 56.11(\mathrm{C}-3), 53.59(\mathrm{C}-6 \mathrm{a}), 53.08\left(\mathrm{~d}, \mathrm{CH}_{3} \mathrm{OP}\right.$, $\left.J_{\mathrm{C}-\mathrm{P}}=6.6\right), 53.00\left(\mathrm{~d}, \mathrm{CH}_{3} \mathrm{OP}, J_{\mathrm{C}-\mathrm{P}}=6.6\right), 45.74(\mathrm{C}-3 \mathrm{a}), 43.16(\mathrm{C}-1), 40.80\left(\mathrm{CH}_{2} \mathrm{P}, J_{\mathrm{C}-\mathrm{P}}=128.3\right)$, 36.49 (C-4), 32.63 (C-2), 32.54 (C-5), HRMS, calc.: [M + 1] 415.01658, found: 415.01584 .

\subsubsection{Synthesis of $\beta$-Ketophosphonate $5 \mathbf{c}$}

The $\beta$-ketophosphonate $\mathbf{5} \mathbf{c}$ was synthesized as in example 7: $93.6 \%$ dimethyl methanephosphonate $(5.03 \mathrm{~g} 100 \%, 40.5 \mathrm{mmol}, 5.4 \mathrm{~mL})$ in anhydrous THF ( $50 \mathrm{~mL}), 1.5 \mathrm{M} n$-butyllitium (27 $\mathrm{mL})$ in hexane, bromo-ester $4 \mathrm{c}(4.46 \mathrm{~g}, 16.2 \mathrm{mmol})$ in THF $(25 \mathrm{~mL})$, acetic acid $(3 \mathrm{~mL})$, TLC (II, $\left.R_{f 4 c}=0.75, R_{f 5 c}=0.21\right)$. The crude product was crystallized from ethyl acetate-hexane, resulting $4.96 \mathrm{~g}(83.3 \%)$ of pure $\beta$-ketophosphonate dimethyl $(2-((2 \mathrm{a} R, 2 \mathrm{a} 1 S, 3 R, 4 \mathrm{a} R, 5 S, 6 \mathrm{a} R)$ 3-bromooctahydro- $1 H$-pentaleno[1,6-bc]furan-5-yl)-2-oxo-ethyl)phosphonate (5c), as puffy needless, mp $75.5-77.0^{\circ} \mathrm{C}$, IR: 2948m, 2856m, 1696s, $1289 \mathrm{~m}, 1211 \mathrm{~s}, 1186 \mathrm{~m}, 1019 \mathrm{vs}, 807 \mathrm{~s}$, 616w, 561m, ${ }^{1} \mathrm{H}-\mathrm{NMR}\left(\mathrm{CDCl}_{3}, \delta \mathrm{ppm}, J \mathrm{~Hz}\right): 4.36$ (m, 2H, H-6, H-5), 3.79 (d, 3H, $\mathrm{CH}_{3} \mathrm{OP}$, 11.2), 3.75 (d, $\left.3 \mathrm{H}, \mathrm{CH}_{3} \mathrm{OP}, 11.2\right), 3.73$ (d, $\left.1 \mathrm{H}, \mathrm{H}-7,8.9\right), 3.54$ (dd, $\left.1 \mathrm{H}, \mathrm{H}-7,5.1,8.9\right), 3.36-3.18$ $(\mathrm{m}, 3 \mathrm{H}, \mathrm{H}-6 \mathrm{a}, \mathrm{H}-3 \mathrm{a}, \mathrm{H}-3), 3.19$ (dd, $\left.1 \mathrm{H}, \mathrm{CH}_{2} \mathrm{P}, J_{\mathrm{H}-\mathrm{P}}=22.6, J_{\text {vic }}=13.7\right), 2.97\left(\mathrm{dd}, 1 \mathrm{H}, \mathrm{CH}_{2} \mathrm{P}\right.$, $\left.J_{\mathrm{H}-\mathrm{P}}=22.6, J_{\mathrm{vic}}=13.7\right), 2.69(\mathrm{dq}, 1 \mathrm{H}, \mathrm{H}-1,5.3,9.4), 2.09-1.95(\mathrm{~m}, 2 \mathrm{H}, \mathrm{H}-2, \mathrm{H}-4), 1.87(\mathrm{dd}, 1 \mathrm{H}$, $\mathrm{H}-4,7.0,14.4), 1.74(\mathrm{dt}, 1 \mathrm{H}, \mathrm{H}-2,10.4,12.9),{ }^{13} \mathrm{C}-\mathrm{NMR}\left(\mathrm{CDCl}_{3}, \delta \mathrm{ppm}\right): 201.14\left(\mathrm{CO}, J_{\mathrm{C}-\mathrm{P}}=\right.$ 3.0), 90.47 (C-6), 74.09 (C-7), 53.60 (C-6a), 55.34 (C-5), 56.18 (C-3), 53.10 (d, $\mathrm{CH}_{3} \mathrm{OP}$, J C-P $=$ 6.1), $53.00\left(\mathrm{~d}, \mathrm{CH}_{3} \mathrm{OP}, J_{\mathrm{C}-\mathrm{P}}=6.1\right), 44.97(\mathrm{C}-3 \mathrm{a}), 43.20(\mathrm{C}-1), 40.50\left(\mathrm{CH}_{2} \mathrm{P}, J_{\mathrm{C}-\mathrm{P}}=128.4\right), 35.13$ (C-4), 32.25 (C-2), HRMS calc. [M + 1] for both isotopes: 367.03045 and 369.02840 , found: 367.02904 and 369.02672 .

\subsection{X-ray Crystallography}

A suitable single crystal sample of $\mathbf{6 c}$ was mounted on a goniometer of the SuperNova diffractometer (Rigaku Corporation, Tokyo, Japan) equipped with dual micro-sources (Mo and $\mathrm{Cu}$ ) and a CCD detector (Eos, Rigaku Corporation, Tokyo, Japan) experimental data being collected with $\mathrm{CuK} \alpha$ radiation, $\mathrm{X}$-ray tube set at $50 \mathrm{kV}$ and $0.8 \mathrm{~mA}$. Data collection strategy, Lorentz-polarization and absorption corrections was conducted with CrysAlis PRO [29] and absorption correction was done using the empirical Multi-scan method which uses spherical harmonics in SCALE3 abs pack algorithm. Crystal structure was solved by direct methods using SHELXS [30] and refined by least square minimization using ShelXL [31], both these programs being implemented in Olex2 (v.21.3) [32] software. All non-hydrogenoid atoms were localized by Fourier difference map and refined anisotropically with the displacement isotropic parameter $\operatorname{Uiso}(\mathrm{H})=1.2 \mathrm{Ueq}(\mathrm{C})$ for all $\mathrm{CH}$ and $\mathrm{CH}_{2}$ groups. Hydrogen atoms were placed in idealized positions and treated as riding as follows: ternary $\mathrm{CH}$ refined with riding coordinates $(\mathrm{C}-\mathrm{H}=0.98 \AA)$ and secondary $\mathrm{CH}_{2}$ refined with riding coordinates $(\mathrm{C}-\mathrm{H}=0.97 \AA)$. By Fourier maps an unassigned electron density is observed around the second site for the carbonyl atom. This disorder was modeled in the sense that the $\mathrm{O} 1$ oxygen of the carbonyl group was located at two positions with occupancy factors 0.69 and 0.31 , but $\mathrm{O} 1$ is linked chemically only to $\mathrm{C} 8$ or $\mathrm{C} 8^{\prime}$. 
For compound $\mathbf{6 b}$ it was not possible to obtain suitable single crystals for the determination of the crystal structure, so an attempt was made to solve the crystal structure by the XRPD method. The methodology applied for determining this structure is presented in ESI (Section 4).

The molecular configuration of the molecules $\mathbf{6 c}$ and $\mathbf{6 b}$ are presented in Figures 3 and 4 in Results and Discussion, and represent asymmetric units, both $\mathbf{6 c}$ and $\mathbf{6 b}$ having a single molecule in the asymmetric unit. The two molecular configurations are similar but not insofar as they can overlap. Each of the two molecules ( $\mathbf{6} \mathbf{c}$ and $\mathbf{6 b}$ ) has six rings, every ring has five members (atoms). These rings adopt approximately a distorted envelope conformation. An ideal envelope conformation has a plane of symmetry that passes through one of the five ring atoms and is perpendicular to the opposite side. In order to estimate the deformation degree of the five member rings related to existence of the symmetry plane, $\Delta$ Cs [33] was defined with the relation and figure below (Figure 5), in which $\Phi_{\mathrm{i}}$ are torsion angles of each ring. Based on the relation below, $\Delta C$ s was calculated for each atom of the rings and those $\Delta \mathrm{Cs}$ with the lowest value were chosen. The results are shown in Table 1. It can be seen that the planes of symmetry pass through the same atoms for molecules $\mathbf{6 c}$ and $\mathbf{6 b}$ with one exception for each molecule: in molecule $\mathbf{6 c}$ the symmetry plane passes through $\mathrm{C}^{\prime}$ and in molecule $\mathbf{6} \mathbf{b}$ the symmetry plane passes through $\mathrm{O}^{\prime}$.

$$
\Delta \mathrm{C}_{\mathrm{s}}=\sqrt{\frac{\sum_{\mathrm{i}=1}^{2}\left(\Phi_{\mathrm{i}}+\Phi_{\mathrm{i}}^{\prime}\right)}{2}}
$$

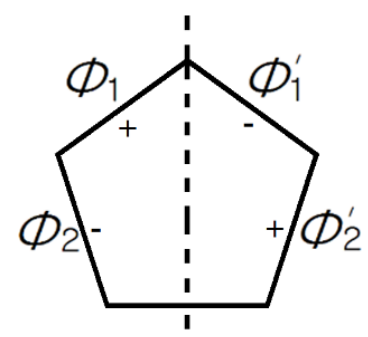

Figure 5. $\Delta$ Cs related torsion angles in five membered rings.

Table 1. Conformational $\Delta C_{s}$ parameter describing the rings $6 \mathbf{c}$ and $\mathbf{6 b}$.

\begin{tabular}{ccccccc}
\hline & $\Delta \mathrm{C}_{\mathrm{s}}$ & $\Delta \mathrm{C}_{\mathrm{s}}$ & $\Delta \mathrm{C}_{\mathrm{s}}$ & $\Delta \mathrm{C}_{\mathrm{s}}$ & $\Delta \mathrm{C}_{\mathrm{s}}$ & $\Delta \mathrm{C}_{\mathrm{s}}$ \\
6c & $(\mathrm{C} 5)$ & $(\mathrm{O} 7)$ & $(\mathrm{C} 3)$ & $\left(\mathrm{C} 4^{\prime}\right)$ & $\left(\mathrm{C} 7^{\prime}\right)$ & $\left(\mathrm{C} 3^{\prime}\right)$ \\
& 12.36 & 15.43 & 5.91 & 14.38 & 14.92 & 5.55 \\
\hline \multirow{3}{*}{$6 \mathbf{b}$} & $\Delta \mathrm{C}_{\mathrm{s}}$ & $\Delta \mathrm{C}_{\mathrm{s}}$ & $\Delta \mathrm{C}_{\mathrm{s}}$ & $\Delta \mathrm{C}_{\mathrm{s}}$ & $\Delta \mathrm{C}_{\mathrm{s}}$ & $\Delta \mathrm{C}_{\mathrm{s}}$ \\
& $(\mathrm{C} 5)$ & $(\mathrm{O} 7)$ & $(\mathrm{C} 3)$ & $\left(\mathrm{C} 4^{\prime}\right)$ & $\left(\mathrm{O} 7^{\prime}\right)$ & $\left(\mathrm{C} 3^{\prime}\right)$ \\
& 5.81 & 9.55 & 3.35 & 9.31 & 15.46 & 1.96 \\
\hline
\end{tabular}

It is worth mentioning that compound $\mathbf{6 c}$ crystallized in the triclinic system P-1 space group with two molecules in the unit cell, having the following lattice parameters: $\mathrm{a}=8.1233 \AA, \mathrm{b}=9.8197 \AA, \mathrm{c}=12.7861 \AA, \alpha=97.873^{\circ}, \beta=100.177^{\circ}, \gamma=95.683^{\circ}$. Compound $\mathbf{6 b}$ belongs to a monoclinic system P21/n space group with four molecules in the unit cell, having the following lattice parameters: $a=5.5121 \AA, b=11.2583 \AA, c=32.2033 \AA, \alpha=90^{\circ}$, $\beta=94.527^{\circ}, \gamma=90^{\circ}$.

CCDC-2068869 (for 6c) and CCDC-2068870 (for 6b) contains the crystallographic data for this contribution. For the compound $6 c$, the prime symbol was replaced with $\mathrm{P}$, for example $\mathrm{C}^{\prime}$ was replaced with $\mathrm{C} 1 \mathrm{P}$. These data can be obtained free of charge via www.ccdc.cam.ac.uk/conts/retrieving.html deposited at 22 June 2021 (or from the Cambridge Crystallographic Data Centre, 12 Union Road, Cambridge CB2 1EZ, UK; fax: (+44)-1223-336-033; or deposit@ccdc.ca.ac.uk). 


\section{Conclusions}

$\beta$-Ketophosphonates with pentalenofurane fragments linked to the keto group were synthesized. Their synthesis started from the pentalenofurane diols 2 and was realized following a sequence of three high yielding steps. Small quantities of secondary compounds $\mathbf{6 b}$ and $\mathbf{6 c}$ were isolated pure and the NMR spectroscopy showed that their structures are esters of the newly formed acids $\mathbf{3 b}$ and $\mathbf{3 c}$ with the starting diols $\mathbf{2 b}$ and $\mathbf{2 c}$. The molecular structures of secondary compounds $\mathbf{6 c}$ and $\mathbf{6 b}$ was confirmed by X-ray crystallography, using two different methods: single crystal X-ray determination method for $\mathbf{6 c}$ and XRPD powder method for $\mathbf{6} \mathbf{b}$. The $\beta$-ketophosphonates $\mathbf{5 a}-\mathbf{5} \mathbf{c}$ will be used as key intermediates for building the $\omega$-side chain of prostaglandins of type III. $\beta$-Ketophosphonate $5 \mathbf{c}$ was already used for obtaining the first $\mathrm{PGF}_{1}$ analogs containing this pentalenofurane scaffold in the $\omega$-side chain of the molecule [28].

\section{Patents}

A patent application [34] describing the synthesis of $\beta$-ketophosphonates having a functionalized pentalenofurane fragment has been submitted to OSIM, Romania.

Supplementary Materials: The following are available online at https:/ / www.mdpi.com/article/ 10.3390 /ijms22136787/s1, NMR-Spectra of the compounds, IR spectra for the compounds 3b, 3c, $4 c, 5 b, 5 c$ and $6 c$; HRMS spectra for the compounds $3 a, 3 b, 3 c, 4 a, 4 b, 4 c, 5 b, 5 c, 6 b$ and $6 c$; X-ray crystallography for the compounds $\mathbf{6 b}$ and $\mathbf{6 c}$ (Table S1: X-ray refinement details from single crystal (a); and XRPD Rietveld refinement (b); Table S2: Distance between atoms ( $\AA$ ) for compounds $6 \mathrm{c}$ and 6b; Table S3: Angles between atoms $\left(^{\circ}\right)$ for compounds $\mathbf{6 c}$ and $\mathbf{6 b}$, Crystal structure determination of compound $\mathbf{6 b}$ from powder diffraction data and Figure $\mathrm{S1}$ ).

Author Contributions: Conceptualization, C.I.T.; methodology, C.I.T. and L.P.; formal analysis, C.D. and M.T.C.; investigation, A.H., M.M., G.B. and E.G.; resources, C.I.T. and A.H.; writingoriginal draft preparation, C.I.T., C.D. and G.B.; writing—review and editing, C.I.T., L.P. and M.T.C.; supervision, C.I.T. and G.B. All authors have read and agreed to the published version of the manuscript.

Funding: We acknowledge the Ministry of Research, Innovation and Digitalization for the grant Orizont-2000, 45/1999/1 and A.H. gratefully acknowledged the University of Bucharest-UniRem project no. 244 and the contract CNFIS-FDI-2020-0355.

Institutional Review Board Statement: Not applicable.

Informed Consent Statement: Not applicable.

Data Availability Statement: Not applicable.

Conflicts of Interest: The authors declare no conflict of interest.

\section{References}

1. Collins, P.W.; Djuric, S.W. Synthesis of Therapeutically Useful Prostaglandin and Prostacyclin Analogs. Chem. Rev. 1993, 93, 1533-1564. [CrossRef]

2. Yankee, E.W.; Axen, U.; Bundy, G.L. Total synthesis of 15-methylprostaglandins. J. Am. Chem. Soc. 1974, 96, 5865-5876. [CrossRef]

3. Crabbe, P.; Fried, J.H. 4,5,13-Prostatrienoic Acid Derivatives. U.S. Patent 3,879,438(A), 25 April 1975.

4. Simons, B.; Dammann, H.-G.; Muller, P.; Orth, D.; Raduntz, H.-E. 13-Thiaprostaglandins Having Cytoprotective Activity. U.S. Patent 4,622,316, 11 November 1986.

5. Kolb, M.; Van Hijfte, L.; Ireland, R.E. A highly convergent synthesis of mexiprestil: 16(R) 16-methoxy 16-methyl PGE 1 methyl ester. Tetrahedron Lett. 1988, 29, 6769-6772. [CrossRef]

6. Skuballa, W.; Raduechel, B.; Vorbrueggen, H.; Elger, W.; Loge, O.; Schillinger, E. Pharmaceutically Active 9-Chloroprostaglandins. U.S. Patent 4,444,788, 24 April 1984.

7. Suga, H.; Konishi, Y.; Wakatsuka, H.; Miyake, H.; Kori, S.; Hayashi, M. Synthesis of 16,16-Dimethyl-Trans- $\Delta^{2}-$ PGE $_{1}$ Methyl Ester (ONO-802). Prostaglandins 1978, 15, 907-912. [CrossRef]

8. Baird, D.T.; Rodger, M.; Cameron, I.; Roberts, I. Prostaglandins and antigestagens for the interruption of early pregnancy. J. Reprod. Fertil. Suppl. 1988, 36, 173-179. 
9. Hayashi, M.; Konishi, Y.; Arai, Y. 6,9-Methano-PGI2 Analogues. U.S. Patent 4,479,966, 30 October 1984.

10. Sodeoke, Y.M.; Ogawa, Y.; Kirio, M. Shibasaki, Stereocontrolled Synthesis of Exocyclic Olefins Using Arene Tricarbonyl Chromium Complex-Catalyzed Hydrogenation. I. Efficient Synthesis of Carbacyclin and Its Analogs. Chem. Pharm. Bull. Tokyo 1991, 39, 309-322. [CrossRef]

11. Kovacs, G.; Simonidesz, V.; Tomoskozi, I.; Kormoczy, P.; Szekely, I.; Papp-Behr, A.; Stadler, I.; Szekeres, L.; Pap, G. A new stable prostacyclin mimic, 7-oxo-PGI2. J. Med. Chem. 1982, 25, 105-107. [CrossRef] [PubMed]

12. Djuric, S.W.; Miyano, M.; Clare, M.; Rydzewski, R.M. A stereocontrolled synthesis of a novel prostacyclin analog "allenecarbacyclin". Application of molecular mechanics calculations to organic synthesis. Tetrahedron Lett. 1987, 28, 299-302. [CrossRef]

13. Schneider, J.; Friderichs, E.; Koegel, B.; Seipp, U.; Stahlberg, H.-J.; Terlinden, R.; Heintze, K. Taprostene sodium. Cardiovasc. Drug Rev. 1993, 11, 479-500. [CrossRef]

14. Aristoff, P.A.; Johnson, P.D.; Harrison, A.W. Total synthesis of a novel antiulcer agent via a modification of the intramolecular Wadsworth-Emmons-Wittig reaction. J. Am. Chem. Soc. 1985, 107, 7967-7974. [CrossRef]

15. Kluge, A.F.; Kertesz, D.J.; O'Yang, C.; Wu, H. Potent prostacyclin analogs based on the bicyclo[4.2.0]octane ring system. J. Org. Chem. 1987, 52, 2860-2868. [CrossRef]

16. Vorbrueggen, H.; Nieuweber, B.; Stuerzebecher, C.S. New Carbacyclines, Preparation thereof and Drug Containing them. WO Patent 86/00895, 13 February 1986.

17. Faustini, F.; Panzeri, A.; Orzi, F.; Di Sale, E.; Ceserani, R. Furyl Derivatives of 16-Substituted Prostaglandins. U.S. Patent 4,585,791, 29 April 1986.

18. Tamura, T.; Inukai, N.; Iwamoto, H.; Yanagisawa, I.; Ishii, Y.; Takagi, T.; Tomioka, K.; Murakami, M. Studies on prostaglandins. VII. Synthesis of prostaglandin derivatives possessing ylidene group on C-16. Yamanouchi Seiyaku Kenkyu Hokoku 1980, 4, 16-29.

19. Hess, H.J.E.; Schaff, T.K. Neue 15-Substituierte-Omega-Pentanorprostaglandine. DE Patent 2,355,731, 22 May 1974.

20. Ngo, V.X.; Old, D.W.; Burk, R.M. 15-Aryl Prostaglandins as EP4 Agonists, and Methods of Use Thereof. U.S. Patent 9,540,357, 10 January 2017.

21. Tănase, C.; Drăghici, C.; Căproiu, M.T. New $\beta$-ketophosphonates for the synthesis of prostaglandin analogues. 1. Phosphonates with a bicyclo[3.3.0]octene scaffold spaced by a methylene group from the $\beta$-ketone. Prostaglandins Leukot. Essent. Fat. Acids 2021 under revision. [CrossRef]

22. Tănase, C.; Căproiu, M.T.; Drăghici, C. New ß-ketophosphonates for the synthesis of prostaglandin analogues. 2. Phosphonates with a bicyclo[3.3.0]octene and bicyclo[3.3.0]octane scaffolds linked to the ß-ketone group. New J. Chem. 2020, 44, 20405-20410. [CrossRef]

23. Tănase, C.; Căproiu, M.T.; Drăghici, C. $\beta$-Ketophosphonates with a Hexahydro- and Octahydropentalene Functionalized Fragment for Obtaining New Prostaglandin Analogs. Patent Pending A/00517, 14 August 2020.

24. Tănase, C.; Cocu, F.; Drăghici, C.; Hanganu, A.; Pintilie, L.; Maganu, M.; Munteanu, C.V.A.; Shova, S. Secondary Compounds in the Catalytic Hydrogenation of Enone and Allylic Alcohol Prostaglandin Intermediates: Isolation, Characterization, X-ray Crystallography. New J. Chem. 2019, 43, 7582-7599. [CrossRef]

25. Tănase, C.; Cocu, F.; Drăghici, C.; Căproiu, M.T. 1,2,3,3a,4,6a-Hexahydro-1,3-pentalenedimethanol, mono and bis OH-Protected Derivatives, Useful Intermediates for Fine Organic Synthesis. Rev. Roum. Chim. 2008, 53, 195-202.

26. Tănase, C.; Drăghici, C.; Shova, S.; Maganu, M.; Cojocaru, A.; Munteanu, C.V.A.; Cocu, F. Regioselective reactions on a 1,3-disubstituted dihydroxymethyl or dicarboxyl hexahydropentalene skeleton F. Tetrahedron 2015, 71, 6852-6859. [CrossRef]

27. Tojo, G.; Fernández, M. Oxidation of Primary Alcohols to Carboxylic Acids, A Guide to Current Common Practice. In Basic Reactions in Organic Synthesis; Springer Science + Business Media, LLC: Berlin/Heidelberg, Germany, 2007 ; pp. 13-31.

28. Tănase, C.; Drăghici, C.; Hanganu, A.; Pintilie, L.; Maganu, M.; Gal, E.; Borodi, G. The First Pentalenofurane Scaffold in the $\omega$-side Chain of a PGF1 Analog. 2021; submitted.

29. CrysAlisPro; Rigaku Oxford Diffraction: Oxfordshire, UK, 2015.

30. Sheldrick, G.M. A Short History of SHELX. Acta Crystallogr. 2008, A64, 112-122. [CrossRef]

31. Sheldrick, G.M. Crystal structure refinement with SHELXL. Acta Crystallogr. 2015, A71, 3-8. [CrossRef]

32. Dolomanov, O.V.; Bourhis, L.J.; Gildea, R.J.; Howard, J.A.K.; Puschmann, H. OLEX2: A complete structure solution refinement and analysis program. J. Appl. Cryst. 2009, 42, 339-341. [CrossRef]

33. Griffin, J.F.; Duax, W.L.; Weeks, C.M. Atlas of Steroid Structure, 2nd ed.; IFI/PLENUM: New York, NY, USA; Washington, DC, USA; London, UK, 1984; p. 8.

34. Tănase, C.; Drăghici, C.; Căproiu, M.T. $\beta$-Ketophosphonates with a Functionalized Pentalenofurane Fragment for Obtaining New Prostaglandin Analogs. Patent Pending A/00516, 14 August 2020. 